\title{
A Graph-Theoretic Model for Time
}

\author{
Paul C. Kainen *'Department of Mathematics, Georgetown University, Washington, DC
}

\begin{abstract}
Time is ordinarily viewed as a path or cycle. Graph models are considered which are a maximal extension of the old ones, subject to a topological constraint. It is shown that the new models have enhanced properties for the representation of temporal phenomena and may provide a useful framework for examining some fundamental assumptions about time.
\end{abstract}

\section{Introduction}

The traditional linear view of time is one-dimensional. Time is taken to be a set of moments (event observations) with intervals (or duration) connecting them in a given sequence. The sequence might be reversible or not, just as a line might be directed or not. Further, the sequence could be finite, infinite at one end, or infinite at both ends.

Hence, it is reasonable to model time by a graph (or digraph) with a set of nodes connected in sequence by edges (or arcs). We shall only discuss the undirected case here. Graph-theoretically, the traditional idea of time is thus well represented by the graph of a finite or infinite path. The periodic case is included by using a cycle-graph instead of a path.

We propose an alternative nonlinear model for time in two dimensions. The significance of planarity in models of time is related to physical ideas which have led to the utilization of complex numbers (i.e., elements of the complex plane) to represent time (see below). Although the elements of our model are the nodes and edges of a graph, which are 0 and 1-dimensional, respectively, the smallest number of dimensions in which the graph can be embedded is 2, while paths and cycles have embeddings in 1-dimensional spaces.

Rather than taking the graph of a point evolving through time as a path in which each node is a snapshot at some instant and each edge is the interval between ticks of the clock, we consider a more densely interconnected graph which adds new adjacencies to the path (and similarly for the cyclic versions). The "cube" of a path makes each instant adjacent not just to its immediate successor and predecessor on the path but also to the moments two and three ticks distant. The choice of three is not arbitrary but is the largest value satisfying the topological constraint of planarity.

The remainder of the paper is organized as follows. Section 2 discusses the issue of representing time as complex numbers which has arisen within physics and mathematics. Section 3 gives an explicit definition of the new model and the next section discusses its properties. Section 5 deals with the cyclic case and Section 6 is a brief discussion of the issue of temporal modeling in the context of Klein's fusion program. Section 7 contains some concluding remarks.

\section{Complex time}

In 1897, Felix Klein used an analysis of the motion of a top to show the advantages of a complexified 4-dimensional analysis in which time was explicitly a complex parameter. His reparameterization of the gyroscopic dynamics problem utilized four parameters $x, y, z, t$ subject to the equation

$$
x^{2}+y^{2}+z^{2}-t^{2}=0 .
$$

Klein specifically denies any "metaphysical significance" for this ([13, pp. 13,18]):

*Computing Anticipatory Systems CASYS'2000, Daniel M. Dubois, Ed., American Institute of Physics Conference Proceedings 573, AIP 2001, pp.490-495 
We shall consider $t$ also as capable of complex values, not for the sake of studying the behavior of a fictitious, imaginary time, but because it is only by taking this step that it becomes possible to bring about the intimate association of kinetics and the theory of functions of a complex variable at which we are aiming.

The resulting non-Euclidean geometry, Klein continues,

... is used solely because it is a convenient method of grouping in geometric form relations which must otherwise remain hidden in formulas.

Since Einstein, the idea of non-Euclidean phenomena is less strange. For example, it has been shown that space and time are not separable in the fashion of a Euclidean geometry. If a clock is taken around the world by airplane, it loses synchrony with an identical clock left on the planet's surface, and the quantitative measure of the effect is predicted by theory. In view of such experimental confirmation of non-Euclidean pheonemena with regard to time, perhaps Klein's analysis should be taken more literally. The idea of using complex numbers seems to have occurred to Klein as an extension of an unpublished method of Jacobi. Use of complex time in the analysis of differential equations was made by Sonia Kovalevsky [1].

Complex numbers correspond to points in the plane. But time has the quality of events separated pairwise by duration. Hence, we think it is natural to model time by means of planar graphs.

\section{The model}

The path is the standard model for time. It can be embedded in the real line. We generalize to a graph embedded in the plane.

The crux of our model is a planar spiral of three turns, extended to a maximal planar graph that is the "cube" (i.e., third-power) of the path. Viewed by itself, a spiral is just a path and so the model we propose includes a copy of the standard model, embedded in a new context and enriched with additional connectivity.

Now we give the formal definition.

For an integer $n \geq 2$ the $n$-path $P_{n}$ is the graph with $n$ vertices corresponding to a partition of some interval into $n-1$ subintervals. Thus, there are $n-1$ edges and each vertex except the two endpoints has degree 2. The length of a path is the number of its edges. Where convenient, we label the vertices $1, \ldots, n$ in the usual order. Graph theory terminology is standard; see, e.g., [7].

For any graph $G$ and nodes $v, w$, let $d(v, w)$ denote the length of a shortest $v-w$-path. For any positive integer $k$, the $k$-th power $G^{k}$ of $G$ is the graph with the same vertices but $v$ and $w$ are adjacent if and only if $d(v, w) \leq k$.

The cube of the $n$-path, $P_{n}^{3}$, is our new model for time. As we shall see, the exponent 3 is not arbitrary and endows the graph with a variety of useful properties. In particular, the cube of a path is maximal planar as we showed with Harary [8]. Here we give a specific parametrization as a particular spiral.

Let $a$ be any positive real number less than 1 and let $\omega$ be the primitive third root of unity, $\omega=$ $(-1+i \sqrt{3}) / 2$.

Then, interpreting 1 as $a^{0} \omega^{0}$, for any nonnegative $s$ and $t$, the sequence of complex numbers of the form

$$
a^{-s} \omega^{-s}, \ldots, a^{-1} \omega^{-1}, 1, a \omega, \ldots, a^{t} \omega^{t}
$$

determines a three-turn spiral embedding in the complex plane of a path of length $s+t$. If each node is joined by a straight line segment to its neighbors in the sequence which are at most 3 steps away, the resulting drawing is a plane graph which is isomorphic to the cube of the path. Note that $\omega^{r}=\omega^{u}$ if and only if $r$ and $u$ are congruent modulo 3. Also, $\omega^{2}$ is the complex conjugate of $\omega$.

The ordinary temporal model may be identified with the action of the integers by translations of the real line. Our new model corresponds to an action of the integers by rotations of the plane by $2 \pi / 3$ (counterclockwise) about zero, with a constant nontrivial contraction or expansion. 


\section{Properties of the model}

The ordinary path is regular of degree 2 except for each end, which is of degree 1 . Similarly, the path-cube $P_{n}^{3}$, for $n \geq 7$, consists of a central portion which is regular of degree 6 , combined with three nodes, at each of the two ends, of degrees $5,4,3$, resp. Thus, both models of time distinguish the boundary of the study period, but the path-cube model has four different gradations rather than two.

The path-cubes have already been used in graph theory. A graph is said to have bandwidth 3 if there exists a labeling of the node set using positive integers so that the labels of adjacent nodes never differ by more than 3. The bandwidth-3 graphs are precisely the subgraphs of the path-cube. In models of program execution, bandwidth relates to transient storage and data reliability requirements.

A graph is $k$-connected if $k$ nodes are sufficient to block every path but $k-1$ are not. The path-cube is 3-connected while the path is only 1-connected; it would be more difficult to disrupt a path-cube. By Menger's theorem, 3-connectedness implies the existence of 3 essentially disjoint paths from 1 to $n$ in $P_{n}^{3}$, and these subgraphs, each approximately one-third the length of the original path, are uniquely determined. For example, $P_{7}^{3}$ contains the union of the paths $(1,2,5,7),(1,3,6,7)$, and $(1,4,7)$.

Extension of time from one to two dimensions allows a much richer local structure of interconnections and is consonant with the view of events proposed by J. H. C. Whitehead. For example, removing a single (non-endpoint) vertex and its two incident edges divides a path into two pieces, while it requires the removal of three consecutive (non-endpoint) vertices and their (up to) 15 incident edges to disconnect the two-dimensional model.

A spanning cycle in a graph is called a Hamiltonian cycle and the graph is said to be Hamiltonian. A graph triangulates a surface if it can be embedded so as to divide the surface into only triangular regions. A triangulation of the $n$-gon is an embedding of $C_{n}$ in the plane with a maximal set of inner diagonals.

For every $n \geq 3, P_{n}^{2}$ is Hamiltonian, as the reader can verify. (For example, for $n=11$, the sequence $1,3,5,7,9,11,10,8,6,4,2$ induces a Hamiltonian cycle.) Since the cube of a graph contains the square as a spanning subgraph, $P_{n}^{3}$ is Hamiltonian.

Because $P_{n}^{3}$ contains $3 n-6$ edges and is planar, it must be a triangulation. Since $P_{n}^{3}$ is also Hamiltonian, it can be embedded as two triangulations of the $n$-gon with no common diagonal. Note that being 3 -connected, the path-cube has a unique embedding in the plane (by Steinitz's theorem). By induction on $n$, the dual graphs of the two triangulated $n$-gons are each paths of length $n-2$.

The dual graph of $P_{n}^{3}$ itself is an interesting polygon which has $n-6$ hexagonal faces and two faces each with 3,4 or 5 sides (not quite Platonic since it has the six non-hexagonal faces). Locally then everything can be symmetric but when a sufficiently large proportion of the dual graph has been explored, some symmetry must break.

Any four consecutive nodes of $P_{n}$ induce a maximal clique (complete subgraph) in $P_{n}^{3}$ and these are the only maximal cliques. Thus, the path-cube of order $n$ has $n-4$ maximal cliques. Joachim Mowitz observed that hence the path-cube looks like the projection onto a plane of a sequence of tetrahedra. If each node is encoded with a complex number, then the path-cube induces a sequence of length $n-4$ with entries which are 8-tuples of real numbers.

Finally, while $P_{n}^{3}$ is planar for all $n$, for $k>3$ by an easy argument based on Euler's formula $P_{n}^{k}$ has genus growing linearly with $n$. Thus, while higher powers have still higher connectivity, they make the topological complexity of the temporal space depend on $n$. Thus, $P_{n}^{3}$ is the largest power of the path which retains a bounded topological complexity independent of the number of vertices.

Even when there are infinitely many vertices, the ordinary path model has no problem with topology. However, for our extension to the path-cube, allowing a spiral in to zero or out to infinity creates an issue of convergence. There will be infinitely many points of the sequence within any neighborhood of zero (or infinity) so the graph cannot be "locally finite" at these two limit points. Thus, the model will need to have a suitably defined topology to handle the infinite case. This may actually be a virtue since it does seem to us that time has a problem with its origin and destination.

Furthermore, for the ordinary path, there is no seeming difficulty in going to the continuum limit - that is, if one assumes one of the various possible ways to define "real" numbers (Dedekind cuts, say). On the other hand, for the path-cube it is not at all clear that there is a continuum limit. This might be feasible to study using the theory of categories applied to continuum mechanics [14].

Another infinite aspect emerges by viewing the present as simultaneously the limit of the past and of the 
future. If the past and future need finer and finer gradations as they approach the present, then we could view the present as "infinity" with respect to the past and "zero" with respect to the future. Topologically, we would need to allow both types of convergence as $\Delta t$ is approaching zero. Perhaps, however, some notion of complex derivative is needed, as Dubois has recently considered [5].

\section{Cyclic time}

For $n \geq 3$, the $n$-cycle $C_{n}$ denotes the unique connected graph which is regular of degree 2 and has $n$ nodes.

There are many cases where time seems to have a cyclic character. For example, there are many cycles with regard to astronomical and biological systems. These can be modeled by a cycle, as in the face of a clock or a chart of the zodiac.

Now we can generalize cyclic time just we did linear time with the cube of a cycle playing the same role as the cube of a path. In fact, by identifying the inner and outermost ends of the canonical spiral embedding of $P_{n+1}$ we obtain a geodesic triangulation of the torus by $C_{n}^{3}([9],[18])$.

The cube of a cycle, our model for periodic time, is regular of degree 6 and 6 -connected. Since the torus is homeomorphic to the space $S^{1} \times S^{1}$ where $S^{1}$ denotes the one-dimensional sphere, the extended model embeds in the cartesian product of the previous model with itself, just as in the case for linear time.

Motivated by examples from psychophysics, we discussed in [10] the idea of "nearly closed" cycles. For example, the visual perception of a nonclosed optical trajectory may be of a closed figure undergoing phase distortion. This amounts to using both periods of the above toroidal temporal space, one representing where in the figure the point is currently, and the other corresponding to the state of the figure in a sequence of states. Nearly closed cycles are also related to approximate commutativity of diagrams, [10], [11].

As a theory of biology develops, we can expect that time should receive a distinct treatment. Above all, biological time is re-entrant and self-referencing. The path-cube and cycle-cube models provide an interesting example of concepts of time developing out of an organic viewpoint.

In particular, we think that the model for time proposed here is in accord with A. N. Whitehead's criteria for a theory of events [19, p. 119]. Notions of hypercycles [6], of systems which anticipate [15] or of hyperincursion [4] might have interesting formulations in the context of our enriched formulation.

\section{The arithmetic of time}

Klein proposed a more broad program than the Erlangen; he called it "fusion". The idea was to somehow unify arithmetic including algebra and analysis, with geometry. In this section, we discuss a connection between Klein's program and our complex model for time.

Of course, Klein's program was designed for applicability to physics. Currently, a group of researchers, largely but not entirely in the U.K., have been exploring the utilization of octonions as a basis for physics, see, e.g., [3], [20], [12]. Their octonion program is certainly a version of Klein's fusion.

The path-cube complex model of time provides a means to complexify each dimension. If one begins with 4 real dimensions, as in a naive model of the world, the cube of a path provides a natural way to complexify each path. But that would lead to an eight-dimensional model. Thus, it might be interesting to find octonionic interpretations.

What I like about the octonions is that they represent an arithmetic which is quite different from real arithmetic in that order and grouping do matter. The failure of commutativity and associativity to hold can have useful consequences. Nonexistence of simultaneity, coming from geometry, means indeterminacy in the order of the multiplicands and in the way in which they are grouped by parentheses. For octonions, this affects the result of multiplication. (Note that while the basis elements are associative up to sign, this is not true for their linear combinations.) Thus, a multiplication can determine topological information.

It would be interesting if this principle is used in biological systems. By "forgetting" the octonionic structure (i.e., taking absolute value), multiplication can easily detect change in magnitude. Combining this with a separate multiplication of octonions should enable parallel detection of change in octonion phase, which is where the topology is.

An octonionic model has another quite interesting property: it forces a break in symmetry because of the geometry of these numbers. The unit integral real numbers and complex numbers have such exceptionally 
banal symmetries that one hardly imagines any reason to iterate the process. But in four dimensions things already get strange, see, e.g., Coxeter [2]. There are 24 unit integer quaternions, and these elements constitute the corners of a particular Platonic polytope in 4 -space. In the case of the octonions, there are 240 vertices in the corresponding figure, which is not one of the three distinct 8-dimensional Platonic polytopes. The polytope does not obey a full set of geometric symmetries.

A path-cube model for time is one possible way to describe the evolution of an octonionic topos. René Thom suggested in [17] that one could replace a point by a cycle to create an enriched fabric. If the urobject is, instead, ineluctibly 8-dimensional, then the octonion geometry could allow novel features in the intertwining of time and space.

\section{Conclusions}

Philosophers have long debated the question of what time really is. This paper does not claim to provide a theory of time. Instead, we have constructed a model with features that may illuminate the underlying phenomenon.

The model proposed here has been shown to have interesting properties. It is intrinsically planar (so events can be interpreted as occurring in complex time) and its connectivity is much richer than the usual onedimensional model. Limits at plus and minus infinity and the issue of continuous vs. discrete measurements are more challenging than in the ordinary model.

Could the three essentially nonoverlapping parallel paths which our 3-connected model provides explain aspects of any natural systems? For instance, one might say that biosystems have can be decomposed into the processes of selection, metabolism and reproduction. In microphysics, there are three fundamental labels or "colors" for the quarks.

Linear time is embedded in our reasoning. Indeed, logic amounts to a kind of universal clock, where theorems mark off the ticks of human (or sentient) development. Mathematics, from this point of view, directs the arrow. A physical schema along these lines has recently been proposed by A. E. Scoville [16].

Time is an implicit part of memory and other aspects of psychology and neuroscience. Linear time does not seem to fit all situations, and the reader can easily recall highly nonlinear models of time in literature (for example, in the work of Jorge Luis Borges and Thomas Pynchon). Perhaps the nonlinear time considered here will be helpful in understanding these psychological aspects of time.

\section{References}

[1] A. V. Borisov and A. V. Tsygvintsev, Kovalevskaya's method in the dynamics of a rigid body, (Russ.), Prikl. Mat. Mekh.61 (1997) no. 1, 30-36; Engl. Transl. J. Appl. Math. Mech., 27-32.

[2] H. M. S. Coxeter, Geometry, Dover, New York, 1999

[3] G. M. Dixon, Division Algebras: Octonions, Quaternions, Complex Numbers and the Algebraic Design of Physics, Kluwer, Dordrecht, 1994.

[4] D. M. Dubois, Introduction of the Aristotle's Final Causation in CAST - Concept and Method of Incursion and Hyperincursion, in Computer Aided Systems Theory - EUROCAST'95, F. Pichler, R. M. Diaz and R. Albrecht, Eds., Springer, Berlin, 1996, pp. 477-493.

[5] D. M. Dubois, Computational derivation of quantum relativist electromagnetic systems with forward-backward space-time shifts, Computing Anticipatory Systems CASYS'99 - Third International Conference. Ed. by D. M. Dubois, American Institute of Physics, AIP Conference Proceedings 517.

[6] M. Eigen and P. Schuster, The Hypercycle, Springer, Berlin, 1979.

[7] F. Harary, Graph Theory, Addison-Wesley, Englewood Cliffs, NJ, 1968.

[8] F. Harary and P. C. Kainen, The cube of a path is maximal planar, Bull. of the Inst. of Combinatorics and its Appl. 7 (1993) 55-56. 
[9] F. Harary, P. C. Kainen and A. Riskin, Every graph of cyclic bandwidth 3 is toroidal, Bull. of the Inst. of Combinatorics and its Appl. 27 (1999) 81-84.

[10] P. C. Kainen, Mathematical cognition, J. of Evolution and Cognition 4 (1998) no. 1, 81-89.

[11] P. C. Kainen, On robust cycle bases, in Proc. 9th Quadrennial International Graph Theory and Combinatorics Conference (GTCC2000), Kalamazoo, Michigan, to appear.

[12] P. C. Kainen, An octonion model for physics, in Proc. of ECHO IV, Odense, 2000, to apear.

[13] F. Klein, The Mathematical Theory of the Top, Charles Scribner's Sons, New York, 1897.

[14] F. W. Lawvere and S. H. Schanuel, Eds., Categories in Continuum Physics, Springer Lecture Notes in Mathematics 1174, Berlin, 1986.

[15] R. Rosen, Life Itself, Columbia University Press, New York, 1991.

[16] A. E. Scoville, The temperature of the universe for $t<t_{p}$, and related properties of the nonmathematical universe, preprint, Aerovest, Inc., Washington, DC, 1999.

[17] R. Thom, Semiophysics: A Sketch, Redwood City, Addison-Wesley, 1991.

[18] C. Thomassen, Five-coloring graphs on the torus, J. Combinatorial Theory B 62(1994) 11-33.

[19] A. N. Whitehead, Science and the Modern World, (Lowell Institute Lectures) MacMillan, New York, 1926.

[20] I. M. Yaglom, Felix Klein and Sophus Lie: Evolution of the Idea of Symmetry in the Nineteenth Century, Birkhauser, Boston, 1988. 